\title{
Predictive Retransmissions for Intermittently Connected Sensor Networks with Transmission Diversity
}

\author{
MD. MAJHARUL ISLAM RAJIB and ASIS NASIPURI, University of North Carolina at Charlotte
}

\begin{abstract}
Batteryless wireless sensor networks that rely on energy harvested from the environment often exhibit random power outages due to limitations of energy resources, which give rise to intermittent connectivity and long transmission delays. To improve the delay performance in such networks, we consider a design strategy that uses predictive retransmissions to maximize the probability of success for each transmission. This is applied to two different transmission diversity schemes: cooperative relaying over unicast routes and opportunistic routing. Performance evaluations from theoretical models and simulations are presented that show that significant gains can be achieved using the proposed approach in such networks.
\end{abstract}

CCS Concepts: • Networks $\rightarrow$ Link-layer protocols; Routing protocols;

Additional Key Words and Phrases: Wireless sensor networks, intermittent connectivity, energy harvesting, retransmission, cooperative relaying, opportunistic routing

ACM Reference format:

Md. Majharul Islam Rajib and Asis Nasipuri. 2017. Predictive Retransmissions for Intermittently Connected Sensor Networks with Transmission Diversity. ACM Trans. Embed. Comput. Syst. 17, 1, Article 12 (September 2017), 25 pages.

https://doi.org/10.1145/3092947

\section{INTRODUCTION}

Limitations of on-board energy resources in wireless sensor nodes continue to be the key challenge that prevents realization of the full potential of wireless sensor networks (WSNs). The requirement of replacing batteries limits the utility of WSNs to the battery life of the nodes. While a lot of research has been focused on energy conservation and management approaches, long-term sustainability of WSNs can only be achieved if the sensor nodes do not have to rely on batteries, which has triggered interest in energy-harvesting technologies for wireless sensor nodes. The goal is to achieve batteryless operation (Minami et al. 2005; Jiang et al. 2005; Dilhac and Bafleur 2014; Shaikh and Zeadally 2016), where nodes harvest energy from the environment and use short-term reliable energy storage devices such as supercapacitors or solid-state batteries. However, a key problem is that most environmental energy sources such as light, mechanical vibrations, radio frequency, heat, and so forth are highly unpredictable and sporadic in nature (Sudevalayam and Kulkarni 2011; Peigney and Siegert 2013). In addition, the amount of energy available from these sources is usually

This work is supported by the National Science Foundation, under grant CNS-1117790.

Authors' addresses: M. M. I. Rajib and A. Nasipuri, Electrical and Computer Engineering Department, University of North Carolina at Charlotte, 9201 University City Blvd., Charlotte, NC 28223; emails: \{mrajib, anasipur\}@uncc.edu.

Permission to make digital or hard copies of all or part of this work for personal or classroom use is granted without fee provided that copies are not made or distributed for profit or commercial advantage and that copies bear this notice and the full citation on the first page. Copyrights for components of this work owned by others than ACM must be honored. Abstracting with credit is permitted. To copy otherwise, or republish, to post on servers or to redistribute to lists, requires prior specific permission and/or a fee. Request permissions from permissions@acm.org.

(C) 2017 ACM 1539-9087/2017/09-ART12 \$15.00

https://doi.org/10.1145/3092947 
limited. The random availability of energy sources coupled with limitations of supply and storage make it difficult to operate the wireless sensor nodes continuously for extended periods of time. Consequently, sensor nodes in such batteryless WSNs may frequently have to shut down their power-hungry components, such as the radio, and go into a deep-sleep mode until they recharge up to a certain level to become active again. This gives rise to intermittent connectivity in WSNs, where the wireless nodes experience random and asynchronous outages ( $\mathrm{Li}$ and Bartos 2014).

Reliable message delivery in such intermittently connected sensor networks (ICSNs) poses several challenges. First, the asynchronous nature of the sleep-awake periods of the nodes makes it difficult to implement fixed scheduling schemes, which is one of the mechanisms that can be applied for achieving reliable data transfer with limited energy wastage. Second, unlike in nonrechargeable sensor networks where reliability is achieved primarily by extending the battery life using energy conservation methods, here more aggressive communication strategies are required that are closely tied to the characteristics of energy harvesting and consumption in the sensor nodes. This is because energy storage devices such as SC and solid-state batteries have low storage and high leakage, leading to depletion of energy even without use. Consequently, achievement of reliable communications in ICSNs requires efficient utilization of the active periods of the nodes that depend on the energy arrival and consumption characteristics.

In this work, we focus on design considerations for reducing the end-to-end transmission delay over multihop routes in severely energy-constrained ICSNs, such as those powered by energy harvested from vibrations and stored using SC or solid-state batteries. We consider two different approaches for achieving this goal.

First, we consider transmission diversity, in which packet transmissions are aided by multiple nodes in the network. We consider two mechanisms for achieving transmission diversity: cooperative relaying over unicast routes and opportunistic routing. Cooperative relaying over unicast routes is described as follows. When a node's transmission attempt fails to reach the desired destination and a common neighbor (neighbor to both source and destination node) overhears the packet, it offers to cooperate by independently forwarding the packet on behalf of the source node. Since nodes go through random sleep and wake cycles, it is more likely that the packet will be delivered faster if more than one node attempts to forward it. This is illustrated in the example depicted in Figure 1. Cooperative relaying improves energy utilization and minimizes delay by tapping into the energy resources of idle nodes in the vicinity of the source and destination, some of which would otherwise be wasted due to ambient processes such as overhearing or leakage. Opportunistic routing is another way of achieving transmission diversity by which any node that receives a packet attempts to forward it to another node that is closer to the destination. The reduction of the transmission delay in this case can be explained similarly to that illustrated in Figure 1, except that here any node closer to the destination can be a forwarder, which results in the packet to be relayed over nodes that wake up early. As discussed in the next section, opportunistic routing has received considerably more attention in the literature compared to unicast routing with cooperative relaying.

Second, we consider optimizing the retransmission interval for minimizing the transmission delay over intermittently connected links. The idea is to determine the best time for a retransmission attempt after each failed transmission that maximizes the probability of successfully reaching a destination. We consider this to be an important parameter since failed retransmissions affect the energy consumption as well as the average delay in the nodes. It is well known that transmission and reception events are the biggest consumers of the energy in low-power wireless sensor nodes. Each energy-harvesting event enables the source to attempt a limited number of transmissions to forward the packet to its parent node. When energy arrival events are infrequent, the retransmission strategy must be based on the energy arrival rate to improve the probability of success. If the 


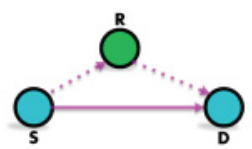

(a)

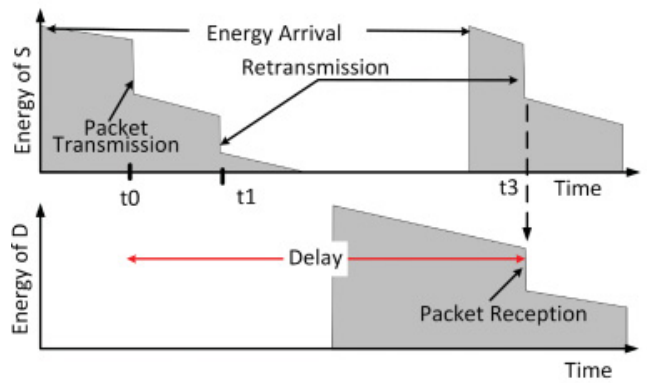

(b)

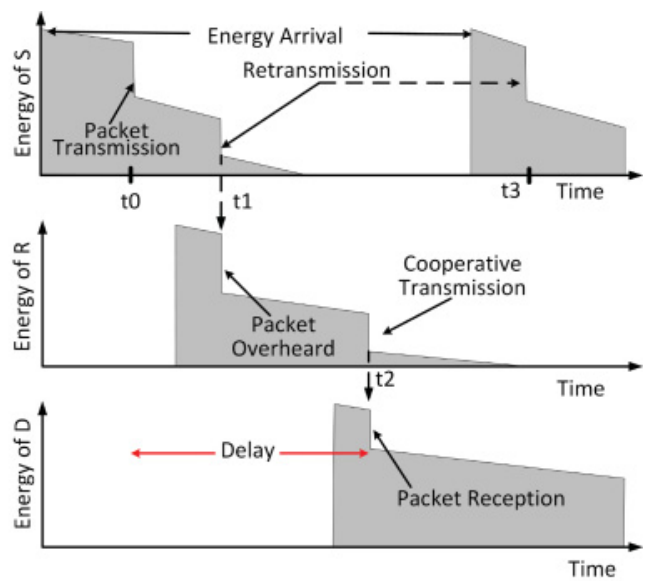

(c)

Fig. 1. Illustration of the reduction of transmission delay in ICSNs using neighbor cooperation. (a) Sample scenario where source $S$ has a packet to transmit to $D$. Node $R$ is a potential cooperator that is in the range of both $S$ and $D$. (b) Variation of energy resources at $S$ and $D$ during transmission without cooperation: when $S$ transmits at $t 0$ and $t 1, D$ does not have enough energy; transmission is eventually successful at $t 3$, resulting in a large delay. (c) Corresponding energy variations at $S, D$, and $R$ when cooperation of $R$ is applied: $S$ 's transmission is overheard by $R$ at $t 1$, which then starts relaying $S$ 's packet and is able to reach $D$ at $t 2$, which is earlier.

retransmission interval is too small, it will result in high energy wastage due to many unsuccessful retransmissions. On the other hand, an excessively high retransmission interval might miss the window of time when the receiver become active and result a longer average delay.

The main contributions of this article are as follows. We first develop a stochastic model to quantify the performance improvement achievable from cooperative relaying. To the best of our knowledge, this is the first attempt to theoretically evaluate the transmission delay over intermittently connected wireless links using cooperative relaying. Second, we present a probabilistic model to find the optimal retransmission interval to reduce the transmission delay over an intermittently connected link. We also develop a mathematical formulation to estimate the expected delay, and use simulations to evaluate the delay characteristics of the proposed model. Finally, we integrate this predictive scheme in both unicast routing with cooperative relaying and opportunistic routing protocols to minimize the end-to-end latency.

The rest of the article is organized as follows. Section 2 discusses related works. A mathematical formulation for analyzing the delay in unicast routing with cooperative relaying is presented in Section 3. In Section 4, we present the formulation of the optimal retransmission interval and analysis of the delay over intermittently connected wireless links. Section 5 discusses the proposed scheme that utilizes optimal retransmission intervals in cooperative relaying and opportunistic routing. In Section 6, we present performance evaluations from computer simulations. Conclusions are presented in Section 7.

\section{RELATED WORK}

Opportunistic routing and cooperative transmission are not entirely new concepts. Here, we discuss related works on these topics that address the transmission delay.

In opportunistic routing, nodes are assumed to be aware of their geographic locations or hop distances from the sink. Any node within a chosen forwarder set that has the potential to forward 


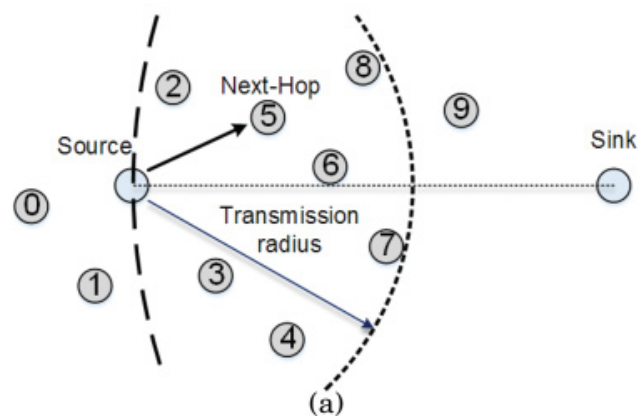

(a)

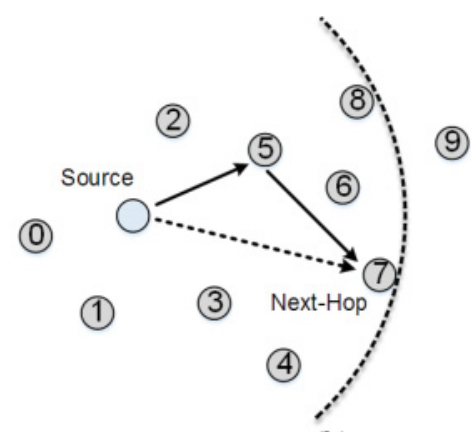

(b)

Fig. 2. Operation of (a) opportunistic routing and (b) unicast routing with cooperative relaying in an intermittently connected network.

the packet closer to the sink forwards it to the next hop. This type of routing opportunistically utilizes available energy in the surrounding nodes. Opportunistic routing has minimal control over the chosen route since there is no way to determine which node in the set is going to be the next hop. For instance, in the illustration in Figure 2(a), a packet transmitted by the source may be forwarded by one or more of nodes 2-8 that make up the forwarder set of the source. Multiple methods have been proposed to choose this forwarder set. For instance, Chen et al. (2009) considered the nodes within a $[-30,30]$-degree angle from the straight line connecting the source to the sink to be the forwarding set. The node within the forwarder set that first receives the packet acknowledges and forwards the packet to the next hop (node 5 in the example). Fafoutis et al. (2015) proposed an opportunistic routing scheme that leverages adaptive duty cycling to find the most eligible forwarder. Here, nodes that have higher energy go through more frequent duty cycling. During a packet transmission, this increases the likelihood of getting a response from a candidate forwarder with higher energy. In contrast, our scheme tries to minimize the number of failed attempts to find the first forwarder before it is selected. Barrabes (2016) suggested a forwarding metric that is based on the neighbor's energy parameters, the forwarder's harvesting rate, and duty cycles. Though this partially considers the energy availability of a route, it has a requirement of getting frequent neighbor information, which is not quite feasible for an intermittently connected network. An opportunistic routing based on the remaining energy and channel state was proposed in Shafieirad et al. (2016). Nodes with higher energy and a better channel state (which are usually ones located close by) get the priority to become the forwarder. One of the concerns with this scheme is that more than one node in the forwarder set may receive the packet, which may result in multiple copies of the same packet to be forwarded to the sink. This might eventually lead to degraded communication performance in the network due to energy wastage, especially in ICSNs, where energy is at a premium. Several approaches have been proposed to overcome this issue. Eu et al. (2010) and Liu et al. (2009) split the forwarder set into a number of regions and assigned a priority to each region based on their potential routing progress. When multiple nodes in the forwarder set receive the packet, nodes from the highest-priority regions declare themselves as next hops first. Nodes from the lower-priority zone discard the packet if they see that a node from a higher-priority zone has already declared itself as the nexthop. However, even with this, the likelihood of forwarding multiple copies is not completely eliminated, since each priority region may consist of multiple nodes. Another approach presented in Landsiedel et al. (2012) requires immediate packet retransmission to inform the forwarding nodes that more than one node has received the packet. 
Cooperative relaying over a unicast route is illustrated in Figure 2(b). Here, every node determines the next hop through some unicast routing protocol, such as least- cost routing. In Figure 2(b), node 7 is the first hop from the source. When the source transmits a packet destined for node 7, neighbors that are common to both the source and node 7 , such as node 5 , assist in reducing the delay through cooperative relaying. In this scenario, if the source's transmission fails to reach 7 but is overheard by 5 (we call it the relay node), node 5 will independently try to forward the packet on behalf of the source. This improves the probability of reaching the destination earlier, thereby reducing the transmission delay from the source to node 7. An automatic repeat request (ARQ) protocol-based cooperative relaying scheme is presented in Tacca et al. (2007) to balance the energy among the neighbor nodes and increase overall throughput. A probabilistic retransmission scheme was proposed in Yoshida et al. (2012) where the optimal number of retransmissions was computed based on charge discharge time and number of potential forwarders. Tutuncuoglu and Yener (2011) presented optimal retransmission selection, leveraging power control and the energy-harvesting rate.

Opportunistic routing and cooperative relaying have their individual benefits. Under similar characteristics of intermittent connectivity, opportunistic routing typically provides lower average transmission delay by invoking higher node diversity. However, cooperative relaying provides several advantages that are beneficial in energy-harvesting sensor networks. First, since cooperative relaying leverages unicast routing, packet duplication is minimized. Therefore, it does not require any additional mechanisms to reduce packet duplications. Next, unicast routing with cooperative relaying allows for more supervised and controlled routing. For example, in the illustration in Figure 2(b), the source may choose node 1 as the next hop to steer packets around nodes $2-8$ if it observes that nodes $2-8$ are getting congested or constrained in energy resources. While the relative performance of cooperative and opportunistic schemes is subject to successful implementations of energy-conserving strategies and network topologies, here, our focus is on improving the delay performance of each respective scheme through the application of predictive retransmission.

\section{ANALYSIS OF DELAY WITH COOPERATIVE RELAYING}

In this section, we analyze the benefits of using cooperative relaying on reducing the transmission delay over an intermittently connected link. In the next section, we present further improvements of the delay performance using predictive retransmissions.

\subsection{Preliminaries}

We consider event-monitoring applications, where nodes periodically sample sensor data and report information to the sink through multihop routing only when specific events of interest are detected. Examples of such applications include environmental condition monitoring, industrial systems monitoring, intrusion detection (Arora et al. 2004), and fire disaster management (Bahrepour et al. 2010), to name a few. It is assumed that at the setup phase of the network, nodes gather route-related data and slowly update the route information through "hello" messages thereafter. We assume that nodes harvest energy from relatively weak environmental energy sources such as mechanical vibrations that occur at independently distributed random instants. Nodes store the harvested energy in SCs and spend it judiciously, giving priority to sensing and processing tasks. When a node's available energy level exceeds a threshold, it activates its radio for transmissions, receptions, and cooperation. Nodes are not time synchronized, which is difficult to achieve in intermittently connected networks. We assume that the energy availability is not sufficient to keep the radio in an active state all the time, resulting in intermittent connectivity of the associated wireless links. In case an event is to be reported, a node utilizes its next available active state to transmit the packet to reach its parent (next hop en route to the sink). In an active state, 
a node engages in a limited number of retransmissions until it receives an acknowledgment from its immediate destination. A random access MAC is assumed with asynchronous duty cycling, such as Low-Power Listening (LPL) (Moss et al. 2007), to conserve energy in its active state.

Though cooperative relaying has the potential of reducing the delay, as stated earlier, it also increases the average energy usage due to a higher number of "radio events" (i.e., transmissions, receptions, and overhearing), leading to shorter average active periods of the nodes. This affects the link connectivity. The effect is exacerbated with an increased number of Cooperator Relays (CRs). Hence, our objective here is to first explore the effect of different system parameters on the delay performance in such networks. To achieve this, we formulate a mathematical model for analyzing the delay performance in a single hop with and without a CR, which is described in the following.

\subsection{Analytical Model}

We consider a single-hop transmission scenario that consists of a source $S$, a cooperator relay $R$, and a destination $D$, as illustrated in Figure 1(a). This can be part of a multihop route. Initially, $S$ contains a data packet that needs to be forwarded to $D$. Each node stores its harvested energy in an energy buffer that has a capacity of $E_{\text {max }}$. The average ambient energy consumption in a node due to leakage, sensing, and processing is denoted by the variable $E_{a}$. We assume that energy arrivals at all nodes occur at random times, which are bursty events typical of mechanical energy harvested on a roadside or bridge obtained from vibrations of passing vehicles. Since such events are largely memoryless, it is reasonable to assume that the interarrival times of energy arrival events are exponentially distributed. For modeling and analysis, we assume a discrete time version of the energy arrival model for which such interarrival times can be described by a geometrical distribution with parameter $P_{e}$. This implies that in the discrete time model, the energy arrival process in each node can be modeled as a Bernoulli process with arrival probability $P_{e}$. The amount of energy in each harvest is represented by a constant $E_{h}$. For the sake of simplicity, we assume that time is slotted and the duration of the slot is sufficiently small so that only one radio event can take place in a slot.

In a larger network, any of the nodes in consideration might overhear transmissions from other nodes in their neighborhood. In ICSNs, all transmissions are dependent on the active periods of the nodes, which depend on energy availability. Since the active periods of different nodes are memoryless, we assume that transmissions from neighboring nodes causing overhearing are random and memoryless. In data collection networks, such as in environmental monitoring applications, the data packets at different nodes are also generated independently. Hence, we model the packet overhearing also by a Bernoulli process, with probability $P_{o} \cdot{ }^{1}$ However, since the ambient data traffic is low in event-monitoring networks, $P_{o}$ should be very low. We assume that the energy consumed by a node in overhearing, transmission, and reception of a packet are the same, represented by $E_{r d}$. This is reasonable, since most low-power radios have similar figures for these events. The probability of a radio event in a node is denoted by $P_{r d}$. Any radio event is assumed to take place at the beginning of a slot and the harvested energy is added to the energy storage at the end of the slot. All acknowledgments are assumed to consume negligible energy and are sent promptly after the reception. When a transmission is unsuccessful, it is repeated with a random delay. This is implemented using a transmissions probability $P_{t x}$ for retransmission in each slot after an unsuccessful attempt.

\footnotetext{
${ }^{1}$ Note that in event-monitoring networks, data traffic in different nodes may have some correlation, but we ignore that in this work for the sake of mathematical tractability.
} 


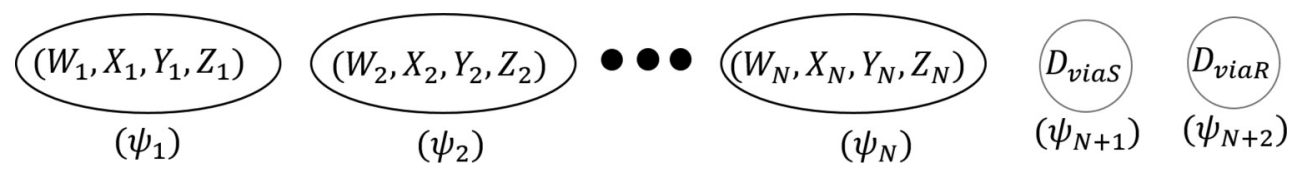

Fig. 3. States of the Markov chain.

With these assumptions, the three-node scenario can be modeled as a four-dimensional Discrete Time Markov Chain (DTMC), where the state is defined by the quadruple $(w, x, y, z)$ as depicted in Figure 3. Here $w \in\{S, S R\}$ indicates whether only the source (denoted as $S$ ) or both the source and relay (denoted as $S R$ ) are currently holding a copy of the packet, and $x$, y or $z \in\left\{m: m \in \mathbb{Z}_{\geq 0} \wedge m \leq\right.$ $\left.E_{\max }\right\}$ denotes the current energy levels of source, relay, and destination, respectively. The packet will be at the source initially, might be copied to the relay, and will reach the destination eventually. Our objective is to find out the required time for the packet to reach the destination.

In order to simplify the analysis of this four-dimensional system, we translate these four variables into a unique single variable $i$ for ease of numerical computations. This is done by setting

$$
\begin{aligned}
i= & w \times\left(E_{\max }+1\right) \times\left(E_{\max }+1\right) \times\left(E_{\max }+1\right) \\
& +x \times\left(E_{\max }+1\right) \times\left(E_{\max }+1\right) \\
& +y \times\left(E_{\max }+1\right)+(z+1) .
\end{aligned}
$$

Here, we denote $w=0$ and 1 to represent the cases $w=S$ and $w=S R$, respectively. This translation leads to $N$ system states where $N=2 \cdot\left(E_{\max }+1\right) \cdot\left(E_{\max }+1\right) \cdot\left(E_{\max }+1\right)$. These states are called transient states. In addition, there are two other states $D_{\text {vias }}$ and $D_{\text {viaR }}$ that represent the state of the system when the destination receives the packet, where the subscript indicates where the packet is received from. These two are absorbing states; that is, when a system moves to any of the these states, it will remain there forever.

We consider that whenever a packet is transmitted either by the source or a relay and the destination is active (i.e., has sufficient energy to receive the packet), it will receive the packet immediately and the system will move from any of the transient states to the absorbing state. Now, the system will experience a transition if and only if any of the four variables in $(w, x, y, z)$ change within a time slot or the destination receives the packet. The values of $x, y$, or $z$ may change in four possible ways corresponding to various combinations of energy events and radio events in each node. To realize this, let's denote $k \in\{x, y, z\}$ as the current value, and $k^{\prime}$ as the value after the transition. Then $k^{\prime}$ and the probability of transitioning into $k^{\prime}$ can be written as

$$
k^{\prime}= \begin{cases}\min \left(k-E_{r d}+E_{i n}, E_{\text {max }}\right), & \text { with } P_{r d} \cdot P_{e} \\ \max \left(k-E_{r d}-E_{a}, 0\right), & \text { with } P_{r d} \cdot\left(1-P_{e}\right) \\ \min \left(k+E_{h}, E_{\text {max }}\right), & \text { with }\left(1-P_{r d}\right) \cdot P_{e} \\ \max \left(k-E_{a}, 0\right), & \text { with }\left(1-P_{r d}\right) \cdot\left(1-P_{e}\right) \\ \text { elsewhere, } & \text { with } 0 .\end{cases}
$$

To find the transition probabilities within the transient states, we first need to get $P_{r d}$ and the possible values of $w$ after transition. Since $P_{r d}$ depends on whether the node has a copy of the packet and how much energy it currently holds, each case must be considered separately. Let us assume that the radio event probability in the source, relay, and destination nodes are denoted by $P_{r d X}, P_{r d Y}$, and $P_{r d Z}$, respectively, and the possible value of $w$ after transition by $w^{\prime}$.

If the current state is described by $(w, x, y, z)$, then the value of $P_{r d X}, P_{r d Y}, P_{r d Z}$, and $w^{\prime}$ can be found as follows: 
Case 1: $z \geq E_{r d}, x \geq E_{r d}$, and $y<E_{r d}$

If $w=S$ or $w=S R$, then $w^{\prime}=w$ with probability $\left(1-P_{t x}\right)$, and $P_{r d X}=P_{r d Z}=P_{o}, P_{r d Y}=0$.

Case 2: $z \geq E_{r d}, x<E_{r d}$, and $y \geq E_{r d}$

If $w=S$, then $w^{\prime}=w$ with probability 1 , and $P_{r d Y}=P_{r d Z}=P_{o}, P_{r d X}=0$.

If $w=S R$, then $w^{\prime}=w$ with probability $\left(1-P_{t x}\right)$, and $P_{r d Y}=P_{r d Z}=P_{o}, P_{r d X}=0$.

Case 3: $z \geq E_{r d}, x \geq E_{r d}$, and $y \geq E_{r d}$

If $w=S$, then $w^{\prime}=w$ with probability $\left(1-P_{t x}\right)$, and $P_{r d X}=P_{r d Y}=P_{r d Y}=P_{o}$.

If $w=S R$, then $w^{\prime}=w$ with probability $\left(1-P_{t x}\right)^{2}$, and $P_{r d X}=P_{r d Y}=P_{r d Y}=P_{o}$.

Case 4: $z \geq E_{r d}, x<E_{r d}$, and $y<E_{r d}$

If $w=S$ or $w=S R$, then $w^{\prime}=w$ with probability 1 , and $P_{r d X}=P_{r d Y}=0, P_{r d Z}=P_{o}$.

Case 5: $z<E_{r d}, x \geq E_{r d}$, and $y<E_{r d}$

If $w=S$, then $w^{\prime}=w$ with probability 1 , and $P_{r d X}=P_{t x}+\left(1-P_{t x}\right) \cdot P_{o}, P_{r d Y}=0, P_{r d Z}=0$.

If $w=S R$, then $w^{\prime}=w$ with probability 1 , and $P_{r d X}=P_{t x}+\left(1-P_{t x}\right) \cdot P_{o}, P_{r d Y}=0, P_{r d Z}=0$.

Case 6: $z<E_{r d}, x<E_{r d}$, and $y \geq E_{r d}$

If $w=S$, then $w^{\prime}=w$ with probability 1 , and $P_{r d X}=P_{r d Z}=0, P_{r d Y}=P_{o}$.

If $w=S R$, then $w^{\prime}=w$ with probability 1 , and $P_{r d Y}=P_{t x}+\left(1-P_{t x}\right) \cdot P_{o}, P_{r d X}=P_{r d Z}=0$.

Case 7: $z<E_{r d}, x \geq E_{r d}$, and $y \geq E_{r d}$

If $w=S$, then $w^{\prime}=S$ with probability $1-P_{t x}$, along with $P_{r d X}=P_{r d Y}=P_{o}, P_{r d Z}=0$, and $w^{\prime}=S R$ with probability $P_{t x}$ along with $P_{r d X}=P_{r d Y}=1, P_{r d Z}=0$.

If $w=S R$, then $w^{\prime}=w$ with probability 1 , and $P_{r d X}=P_{r d Y}=P_{t x}^{2}+2 \cdot\left(1-P_{t x}\right)+\left(1-P_{t x}\right)^{2}$. $P_{o}, P_{r d Z}=0$.

When cooperation is not applied, potential cooperators should not retain a copy of the packet to forward on behalf of the source. That is, the system will never move from $w=S$ to $w=S R$. Under this situation:

If $w=S$, then $w^{\prime}=S$ with probability 1 , and $P_{r d X}=P_{t x}+\left(1-P_{t x}\right) \cdot P_{o}, P_{r d Z}=0$.

Case 8: $z<E_{r d}, x<E_{r d}$, and $y<E_{r d}$

If $w=S$ or $w=S R$, then $w^{\prime}=w$ with probability 1 , and $P_{r d X}=P_{r d Y}=P_{r d X}=0$.

With these, the probability of moving from a transient state $S_{i}$ to $S_{j}$ can be written as follows:

$$
P_{i, j}=P_{w_{i}, w_{j}} \times P_{x_{i}, x_{j}} \times P_{y_{i}, y_{j}} \times P_{z_{i}, z_{j}}
$$

where $S_{i}$ can be decomposed into quadruple $\left(w_{i}, x_{i}, y_{i}, z_{i}\right)$, and $S_{j}$ into $\left(w_{j}, x_{j}, y_{j}, z_{j}\right) . P_{x_{i}, x_{j}}$ denotes the probability of moving from $x_{i}$ to $x_{j}$. All the probabilities required for this equation can be found from Equation (2) and the cases described previously.

We now construct the state transition matrix $P$, where the element $P_{i, j}$ in the $i^{\text {th }}$ row and $j^{\text {th }}$ column represents the state transition probability. We divide the state space of the DTMC into two subsets: one containing all the transient states and another the two absorbing states. We can represent the transitions in $P$ as

$$
P=\left[\begin{array}{ccc|cc}
P_{1,1} & P_{1,2} & \cdots & P_{1, N+2-1} & P_{1, N+2} \\
P_{2,1} & P_{2,2} & \cdots & P_{2, N+2-1} & P_{2, N+2} \\
\vdots & \vdots & \vdots & \vdots & \vdots \\
\hline 0 & 0 & \cdots & 1 & 0 \\
0 & 0 & \cdots & 0 & 1
\end{array}\right] .
$$




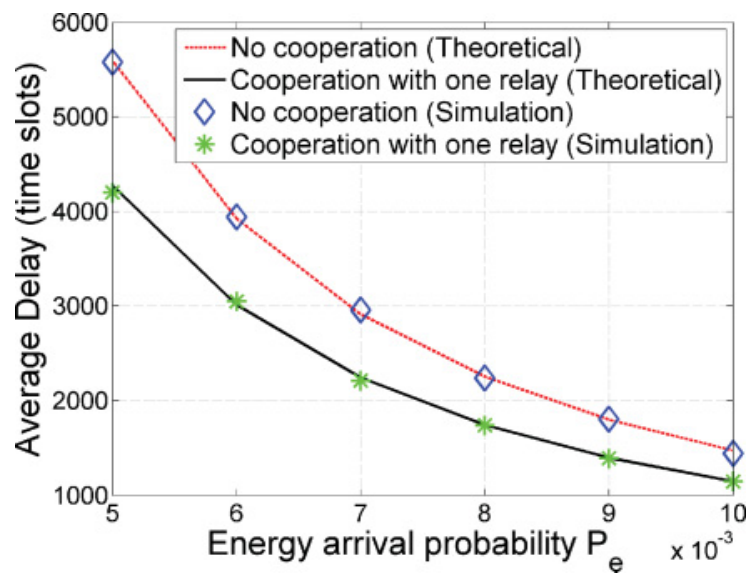

Fig. 4. Comparison between theoretical model and simulation.

If we denote the upper left partition of the matrix by $T$, the upper right by $U$, and the lower right by $A$, then we may express the matrix $P$ by

$$
P=\left[\begin{array}{l|l}
T & U \\
\hline 0 & A
\end{array}\right] .
$$

To find the expected delay before the packet gets delivered to the destination, it is necessary to find the expected time that the system spends before moving into any of the absorbing states. This can be found from the following equation (Stewart 2009):

$$
D=(I-T)^{-1} \cdot e,
$$

where $e$ is a column matrix of ones. Here, the $i^{\text {th }}$ element of $D$ provides the mean time to absorption from state $i$.

Numerical results obtained using this model on the average delay over a wireless link with and without cooperative relaying are plotted in Figure 4. The figure depicts the variation of the average delay with $P_{e}$, where the overhearing probability $P_{o}$ is fixed at 0.0001 per slot, and all other parameters are as listed in Table 1 . These results clearly depict that cooperative relaying reduces the average delay, which is due to transmission diversity. The delay improvement is particularly pronounced at low energy arrival rates (i.e., when the link connection is more intermittent). The theoretical results are validated with those obtained from computer simulations written in $\mathrm{C}++$. While these results are encouraging, it must be noted that these were obtained for a constant radio event probability. In reality, cooperative relaying will result in additional radio activity that will depend on the network scenario and event probabilities and needs to be evaluated using a full-scale network model.

\section{PREDICTIVE RETRANSMISSION FOR INTERMITTENT WIRELESS LINKS}

We now describe the basis for the proposed probabilistic predictive retransmission scheme to further reduce the transmission delay. We first demonstrate that the probability of success in an intermittently connected wireless link can be maximized by the selection of an optimum retransmission interval that depends on the available energy level at the node (battery life) and the energy-harvesting rate. We then show that using the optimum retransmission interval for every retransmission attempt minimizes the average packet transmission delay over the intermittently connected wireless link. The idea is illustrated in Figure 5. In Figure 5(a), the transmitter employs 
Table 1. Parameters Used in the Analysis for Cooperative Relaying

\begin{tabular}{|c||c|}
\hline Energy storage capacity, $E_{\max }$ & 20 \\
\hline Energy consumed in a radio event, $E_{r d}$ & 10 \\
\hline Amount of energy in each harvest, $E_{h}$ & 20 \\
\hline Average ambient energy consumption, $E_{a}$ & 1 \\
\hline Probability of overhearing, $P_{o}$ & 0.0001 \\
\hline Transmit probability in each slot, $P_{t x}$ & 0.1 \\
\hline
\end{tabular}

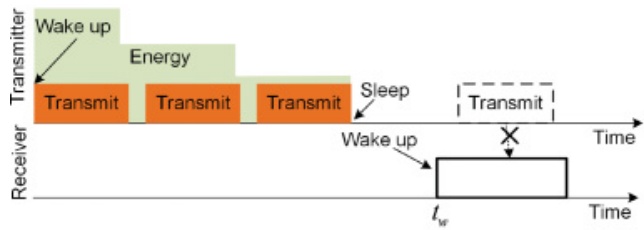

(a)

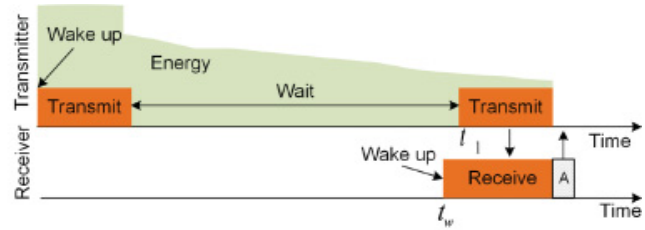

(b)

Fig. 5. Illustration of (a) back-to-back and (b) predictive retransmissions.

back-to-back retransmissions, where the expectation is for the receiver to receive and acknowledge the packet whenever it wakes up. However, as illustrated in Figure 5(a), this might lead to a situation where the transmitter depletes its energy before it reaches the receiver. We propose that instead of performing immediate retransmissions, it is more effective for the transmitter to retransmit at a time when the probability of the receiver waking up is predicted to be maximum (see Figure 5(b)). This reduces wasteful retransmissions and maximizes the probability of successful transmission in the transmitter's current active period, thereby reducing the risk of the transmitter going back to sleep prior to successfully transmitting the packet.

\subsection{Energy Dynamics of a Sensor Node}

In order to predict the optimal retransmission time, it is essential to characterize the energy dynamics of a sensor node. The basic energy expenditure and harvesting behavior of a node is the same as described in Section 3.2. Based on these assumptions, we can conclude that the energy stored in a sensor node's storage device changes due to primarily three causes:

-First, the energy level increases by $E_{h}$ for each "energy arrival" event as discussed previously.

- Second, the energy gets depleted due to radio events, which include packet transmissions (consumes $E_{t}$ ), receptions (consumes $E_{r}$ ), or overhearing (consumes $E_{o}$ ).

-Finally, we consider that when a node is in active state, ambient events such as sensing, processing, and low-power listening deplete its energy at a constant rate. To simplify, we term all these activities together as "ambient activities," which require an average "ambient consumption” of $E_{a}^{t}$ or $E_{a}^{n t}$ energy for transmitter and nontransmitter, respectively. This spans throughout the active period.

Variations in a node's energy level due to these activities are illustrated in the top of Figure 6. When the energy is depleted to such a level that a node can no longer participate in transmissions or receptions, it goes into a deep sleep or inactive mode. Due to low and random energy availability, the length of the inactive period is assumed to be large compared to the active periods and no node can schedule sleep-wake cycles ahead of time. 


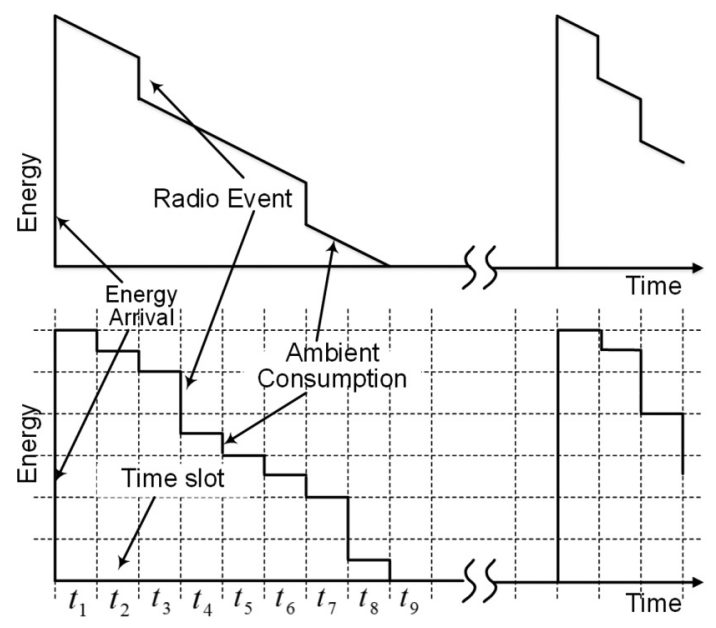

Fig. 6. Continuous time representation (top) and its discrete time approximation (bottom) of the variation of energy level in a rechargeable sensor node.

Table 2. Notations Used

\begin{tabular}{|l|l|}
\hline$E_{h}$ & Amount of energy in each harvest \\
\hline$E_{c}$ & Energy remaining in the storage \\
\hline$E_{t}$ & Required energy for a packet transmission \\
\hline$E_{r}$ & Required energy for a packet reception \\
\hline$E_{o}$ & Energy consumed in overhearing \\
\hline$E_{a}^{t}$ & Ambient consumption by transmitting node \\
\hline$E_{a}$ & Ambient consumption of a general node \\
\hline$P_{e}$ & Energy arrival probability \\
\hline$P_{o}$ & Probability of overhearing \\
\hline$P_{c}$ & Probability that the channel is error free \\
\hline$T_{w}$ & Waiting time before retransmission \\
\hline
\end{tabular}

To make computations tractable, we consider a discrete time version of this continuous time model (bottom of Figure 6). We assume that time is slotted and each slot is sufficiently small so that only one transmission can take place in a slot. The interval between two successive energy arrival events can then be represented by a geometric distribution with a parameter $P_{e}$. Considering that the energy arrival rate is small (i.e., the interenergy arrival times are long), the probability of occurrence of another energy arrival event while a node is still active is negligible. For simplification, we assume that all energy-related events, such as harvesting and consumption, occur at the end of the time slot. Since all nodes perform LPL, any node can capture the transmission of a nearby node as long as it is active. This is described as overhearing (having a probability parameter $P_{o}$ ). With these considerations, we formulate the optimal waiting time before retransmission in the following section. A summary of the notations used is presented in Table 2.

\subsection{Optimal Retransmission Interval}

Once a transmission is unsuccessful (due to the fact that the destination is inactive), a successful rendezvous between active states of a transmitter and receiver requires that the transmitter remains active while the destination wakes up from the sleep state and remains reception capable 


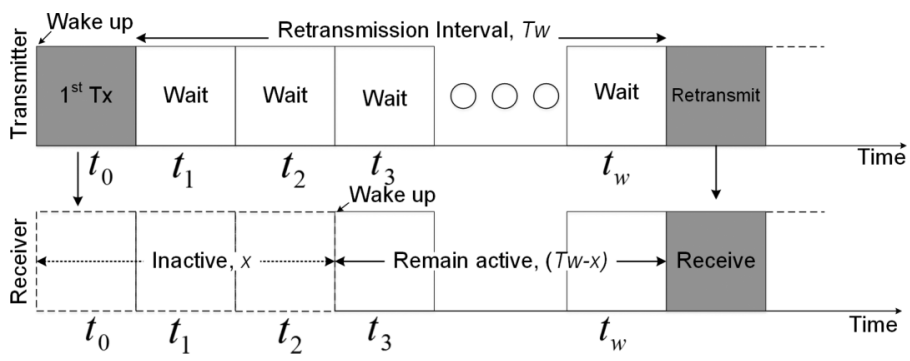

Fig. 7. Finding the optimal retransmission interval.

(i.e., has sufficient energy to receive) for at least up to the point where the transmitter retransmits. In the following, we develop each of these probabilities for the back-to-back transmissions and using a retransmission interval of $T_{w}$ after a failed transmission attempt.

Probability that transmitter remains active: To find the success probability of a retransmission at a random time, we first find the probability that the source remains active up to that time. Let's consider Figure 7, where a transmitter node wakes up from the inactive state at $t_{0}$ with $E_{h}$ amount of energy. After waking up, it immediately performs a transmission, which is unsuccessful. The transmitter node is now left with $E_{c}=E_{h}-E_{t}$ amount of energy. If the node decides to wait for an interval $T_{w}$ before the next retransmission, it has to have at least $E_{c}-E_{t}$ energy by the end of $T_{w}$ waiting period to perform another transmission. Now, if the transmitter overhears $\eta$ packets within $T_{w}$, the following inequality must hold in order for it to be able to retransmit at $\left(T_{w}+1\right)$ :

$$
\begin{aligned}
& \eta E_{o}+\left(T_{w}-\eta\right) E_{a}^{t} \leq\left(E_{c}-E_{t}\right) \\
& \text { or, } \eta \leq \frac{\left(E_{c}-E_{t}\right)-T_{w} E_{a}^{t}}{\left(E_{o}-E_{a}^{t}\right)} .
\end{aligned}
$$

The maximum amount of overhearing that it will be able withstand and still remain capable of retransmission is

$$
\eta_{m}^{t}=\left\lfloor\frac{\left(E_{c}-E_{t}\right)-T_{w} E_{a}^{t}}{\left(E_{o}-E_{a}^{t}\right)}\right\rfloor .
$$

Now, the probability of remaining capable of transmission after $T_{w}$ is given by the probability of overhearing at most $\eta_{m}^{t}$ packets within $T_{w}$, which is

$$
P_{w}^{t}\left(T_{w}\right)=\sum_{i=0}^{\eta_{m}^{t}}\left(\begin{array}{c}
T_{w} \\
i
\end{array}\right) P_{o}^{i}\left(1-P_{o}\right)^{T_{w}-i}
$$

Probability that receiver is active: In order for the retransmission to be successful, it is also required that the receiver wakes up in the mean time and remains capable of reception until the time of retransmission. The probability that the receiver wakes up after $x$ (see Figure 7) is $P_{e}(1-$ $\left.P_{e}\right)^{(x-1)}$. After waking up, the probability that it remains capable of reception from $x$ to $T_{w}+1$ and beyond can be expressed as

$$
P_{w}^{r}\left(T_{w}+1-x\right)=\sum_{i=0}^{\eta_{m}^{r}}\left(\begin{array}{c}
T_{w}+1-x \\
i
\end{array}\right) P_{o}^{i}\left(1-P_{o}\right)^{T_{w}+1-x-i},
$$

where

$$
\eta_{m}^{r}=\left\lfloor\frac{\left(E_{h}-E_{r}\right)-\left(T_{w}+1-x\right) E_{a}^{n t}}{\left(E_{o}-E_{a}^{n t}\right)}\right\rfloor
$$


4.2.1 Probability of Success with Predictive Retransmission Interval. The probability of a successful retransmission after an interval $T_{w}$ is then given by the product of the probabilities of the source remaining capable of transmission up to that time and that the receiver wakes up prior to $T_{w}$ and remains capable of reception afterward. In summary, the probability of a successful retransmission is obtained as

$$
P_{w}^{s}\left(T_{w}\right)=P_{w}^{t}\left(T_{w}\right) \sum_{x=1}^{T_{w}+1}\left[P_{e}\left(1-P_{e}\right)^{(x-1)} P_{w}^{r}\left(T_{w}+1-x\right)\right] P_{c} .
$$

Therefore, the maximum success probability achievable with the next retransmission is

$$
P_{m}^{p}=\max _{T_{w}} P_{w}^{s}\left(T_{w}\right) \text { s.t. } 0 \leq T_{w} \leq\left\lfloor\frac{\left(E_{c}-E_{t}\right)}{E_{a}^{t}}\right\rfloor .
$$

Consequently, the optimum retransmission interval that maximizes the probability of a successful retransmission is given by

$$
T_{o}^{p}=\arg \max _{T_{w}} P_{w}^{s}\left(T_{w}\right) .
$$

The optimum solution $T_{o}^{p}$ depends on the parameters listed in Table 2, which include fixed parameters as well as node-specific and environmental parameters. While the fixed parameters depend on the hardware platform used, node-specific parameters such as the energy arrival and overhearing probabilities, the amount of energy in each harvest, and the current energy level may be estimated by the nodes. To avoid extensive computations, each node may obtain $T_{o}^{p}$ from lookup tables that are computed offline based on a select set of fixed parameters and a range of nodespecific dynamic parameters. Note that this requires mostly static parameters and a small number of dynamic parameters, most notably the current energy level.

To determine the effect of different node parameters on the optimum retransmission interval, we plot the variations of the probability of successful retransmission with a retransmission interval $T_{w}$ for different sets of parameters in Figure 8. The default parameters for these figures are provided in Table 3. Also, since the energy arrival probability is quite low, we approximate the Binomial distributions of Equations (9) and (10) with a Poisson distribution. We also consider $P_{c}=1$ to focus mainly on parameters related to the sensor nodes.

In Figure 8(a), we study the effect of the ambient energy consumption on the optimal retransmission interval. It is observed that the probability of success for retransmissions generally increases with increasing values of $T_{w}$. This is due to the fact that a longer interval allows more likelihood of the receiver becoming active in the meantime. However, if the retransmission interval becomes too long, the success probability sharply falls since the transmitter loses energy from ambient activities. It must be noted that when the ambient energy consumption is high, the probability of success is lower. Figure 8 (b) suggests a smaller waiting period between successive retransmissions if harvested energy is low.

A general observation from these two figures is that the probability of success is highest for the longest possible retransmission interval; that is, it is better to wait until only enough energy for a single transmission is left. For example, if we assume that a node currently has 20 units of energy, each radio event consumes 5 units of energy, and ambient activities constantly consume 1 unit of energy, the node should wait for 15 time units before the next retransmission. This maximizes the chances for the receiver to become active while the source still has energy to transmit. However, this observation does not apply for all the cases. For example, Figure 8(b) depicts that for $E_{h}=$ 40, the optimal waiting time becomes 27 instead of 30. In Figure 8(c), we see the variations of the optimal retransmission interval with respect to different energy arrival probabilities. Here, a small increase in energy arrival probability has little effect on the optimal delay time; however, the 


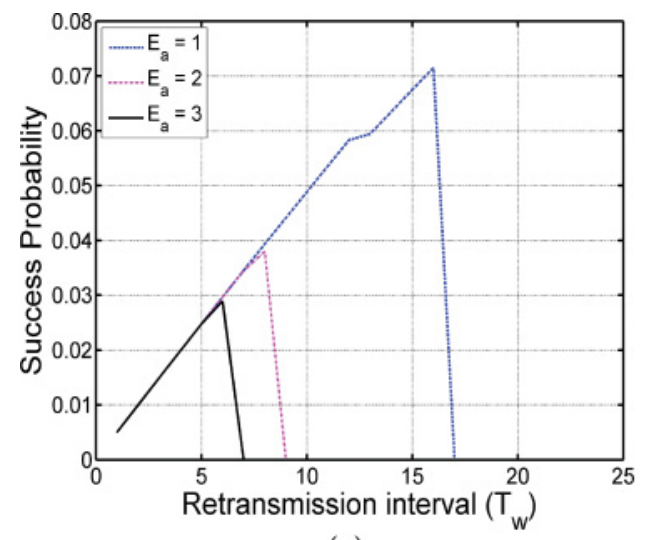

(a)

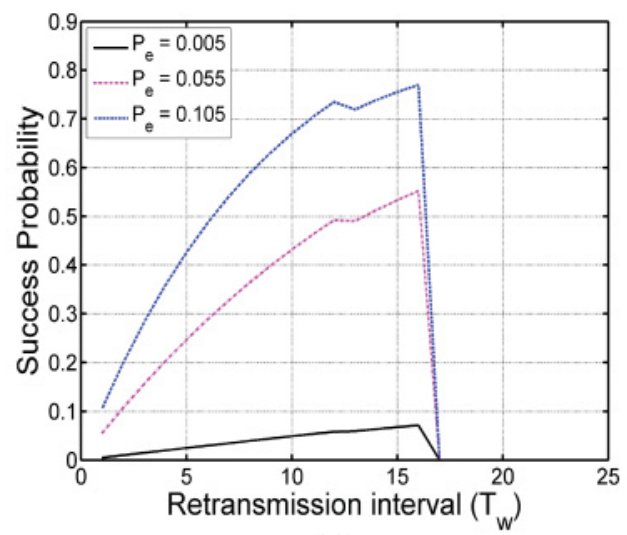

(c)

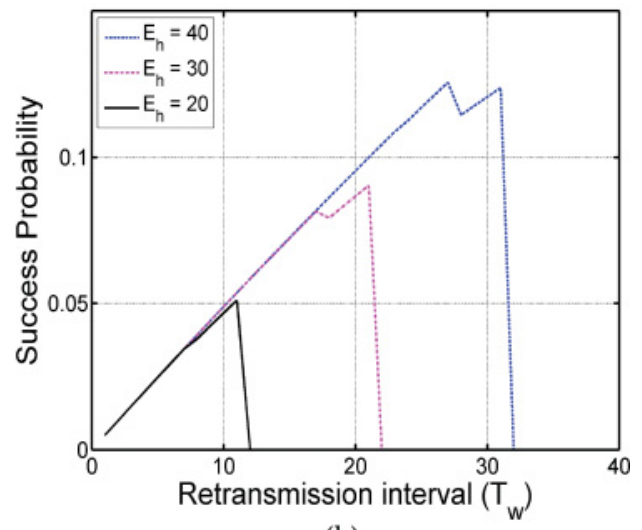

(b)

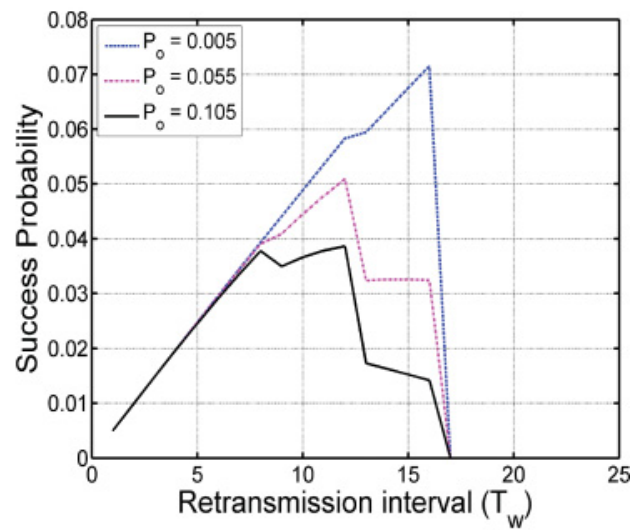

(d)

Fig. 8. Probability of success of packet retransmissions in an intermittently connected wireless link using different retransmission intervals.

Table 3. Simulation Configuration

\begin{tabular}{|l|c|}
\hline Amount of energy in each harvest, $E_{h}$ & 25 \\
\hline Required energy for a radio event, $E_{r}$ & 5 \\
\hline Energy consumption from ambient activities, $E_{a}$ & 1 \\
\hline Probability of an error-free channel, $P_{c}$ & 1 \\
\hline Probability of overhearing, $P_{o}$ & 0.005 \\
\hline Energy arrival probability, $P_{e}$ & 0.005 \\
\hline
\end{tabular}

success probability is vastly improved. This is due to the fact that a higher waiting time ensures a higher likelihood of the receiver becoming active regardless of the energy arrival probability. Figure $8(\mathrm{~d})$ indicates that the success probability significantly decreases with higher $P_{o}$, which indicates the detrimental effect of higher overhearing.

4.2.2 Probability of Success with Back-to-Back Retransmissions. To perform a comparison, we now obtain the maximum success probability associated with back-to-back retransmissions. Given that the first transmission is not successful, if the maximum number of transmission attempts 


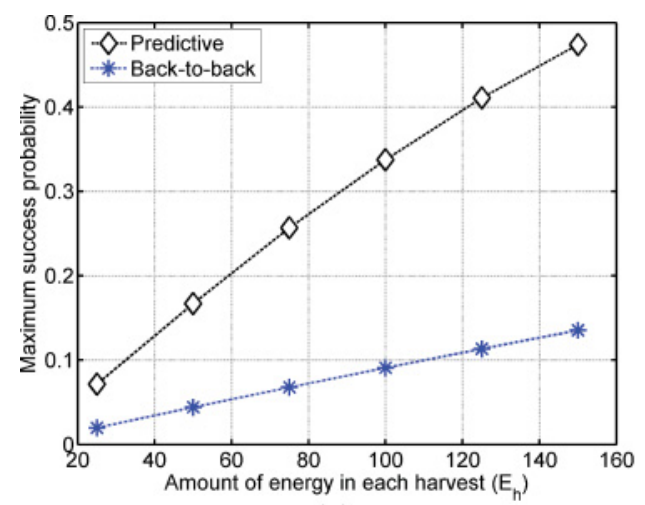

(a)

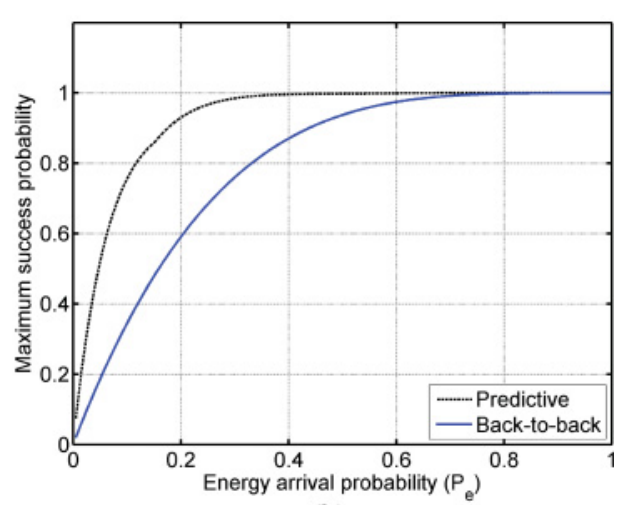

(b)

Fig. 9. Maximum success probability achievable using predictive and back-to-back retransmissions in an intermittently connected wireless link.

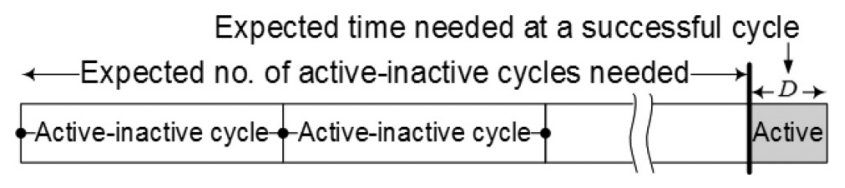

Fig. 10. Calculation of the packet transmission delay.

possible after waking up from an inactive state with $E_{h}$ energy is denoted by $\beta+1$, then we can write

$$
\beta=\left\lfloor\frac{\left(E_{h}-E_{t}\right)}{E_{t}}\right\rfloor
$$

Here, the success probability for each retransmission attempt depends on whether the receiver underwent an energy arrival event in the previous slot and also on the channel condition. The cumulative success probability for retransmissions can therefore be written as

$$
P_{m}^{b}=\sum_{i=1}^{\beta} P_{e} P_{c}\left(1-P_{e} P_{c}\right)^{i-1} .
$$

We plot the maximum attainable success probability using back-to-back retransmissions and that using the proposed predictive retransmission scheme in Figure 9. It is clear that the predictive retransmission strategy offers a higher probability of success compared to the back-to-back transmission policy by leveraging the statistical information at the time of the first transmission and adjusting the retransmission interval accordingly. Moreover, the back-to-back retransmission is a special case for the predictive retransmission scheme when $T_{w}=0$ provides the highest probability of success.

\subsection{Delay Analysis}

We now evaluate the average transmission delay over an intermittently connected wireless link using the two retransmission schemes. Our approach is to first determine the probability that a packet transmission will be successful in a single active-inactive cycle of the source node. As illustrated in Figure 10, the total transmission delay is equal to the period of time covering the 
expected number of active-inactive cycles required before the packet transmission is successful plus the expected delay involved within the active period where the packet is delivered.

The overall probability of success for transmitting a packet in a single active-inactive cycle depends on the probability of success at the very first transmission after waking up from sleep state, and that from retransmission, if the first transmission is not successful. In the following, we find the success probability of the first transmission, the maximum success probability in a single active-inactive period that is achievable using each retransmission policy, and the expected delay involved within an active period to obtain the overall delay.

The success probability of the very first transmission attempt after waking up from a long sleep period depends on whether the receiver is active or not during that transmission. Hence, we find the active probability of the receiver at any random slot.

Let's consider that the receiver has enough energy to be in the active period for an average $\left(\mu_{a}+1\right)$ units of time. Within this time, if an average of $\alpha$ overhearing occurs, we get $\mu_{a} \cdot P_{o}=\alpha$ or $\mu_{a}=\frac{\alpha}{P_{o}}$. Using a similar approach as Equation (7), we may write

$$
\begin{aligned}
\alpha E_{o}+\left(\mu_{a}-\alpha\right) E_{a} & =E_{h}-E_{r} \\
\text { or, } \alpha\left(E_{o}-E_{a}\right)+\mu_{a} E_{a} & =E_{h}-E_{r} \\
\text { or, } \mu_{a} \cdot P_{o}\left(E_{o}-E_{a}\right)+\mu_{a} E_{a} & =E_{h}-E_{r} \\
\text { or, } \mu_{a} & =\frac{E_{h}-E_{r}}{P_{o}\left(E_{o}-E_{a}\right)+E_{a}} .
\end{aligned}
$$

Since the time between successive energy arrivals is geometrically distributed with parameter $P_{e}$, the average interval is $\mu_{e}=\frac{1}{P_{e}}$. When $\mu_{a}$ is much smaller than $\mu_{e}$ (as in the case of an intermittently connected network), the probability that the destination is active at a random time slot is approximately $\frac{\left(\mu_{a}+1\right)}{\mu_{e}}$. Hence, the probability of success at the very first transmission can be written as

$$
P_{1} \approx\left[\frac{\left(\mu_{a}+1\right)}{\mu_{e}}\right] P_{c} .
$$

Using Equations (13), (16), and (18), we can write the overall success probability at any activeinactive cycle associated with predictive retransmission as

$$
P_{S P}=P_{1} P_{c}+\left(1-P_{1} P_{c}\right) P_{m}^{p}
$$

If a transmission is successful in an active period, the expected time required for the success from the beginning of that active period can be written as

$$
E_{p}[D]=\frac{1}{P_{S P}}\left(P_{1} P_{c}+\left(1-P_{1} P_{c}\right) P_{m}^{p}\left(T_{o}^{p}+1\right)\right) .
$$

Similarly, the overall success probability associated with back-to-back retransmissions is

$$
\begin{aligned}
P_{S B}= & P_{1} P_{c}+\left(1-P_{1} P_{c}\right)\left(P_{e} P_{c}\right)+\cdots \\
& +\left(1-P_{1} P_{c}\right)\left(1-P_{e} P_{c}\right)^{\beta-1}\left(P_{e} P_{c}\right) \\
= & P_{1} P_{c}+\left(1-P_{1} P_{c}\right) P_{m}^{b} .
\end{aligned}
$$


Consequently, the expected time required for success from the beginning of an active period is

$$
\begin{aligned}
E_{b}[D]= & \frac{1}{P_{S B}}\left(P_{1} P_{c}+2\left(P_{e} P_{c}\right)\left(1-P_{1} P_{c}\right)+\cdots\right. \\
& \left.+\left(1-P_{1} P_{c}\right)\left(1-P_{e} P_{c}\right)^{\beta-1}\left(P_{e} P_{c}\right)(\beta+1)\right) \\
= & \frac{1}{P_{S B}}\left(P_{1} P_{c}+\sum_{m=2}^{\beta+1} m\left(P_{e} P_{c}\right)\left(1-P_{e} P_{c}\right)^{m-2}\left(1-P_{1} P_{c}\right)\right) .
\end{aligned}
$$

The length of an average active-inactive cycle is equivalent to the average energy arrival interval $\mu_{e}$. If we denote the overall success probability at any cycle in general by $P_{s}$ and the expected delay at a successful cycle by $\bar{D}$, we can express the expected overall delay as

$$
\begin{aligned}
E[D]= & \bar{D} P_{s}+\left(\bar{D}+\mu_{e}\right) P_{s}\left(1-P_{s}\right) \\
& +\left(\bar{D}+2 \mu_{e}\right) P_{s}\left(1-P_{s}\right)^{2}+\cdots \\
= & \bar{D} P_{s}+\bar{D} P_{s}\left(1-P_{s}\right)+\bar{D} P_{s}\left(1-P_{s}\right)^{2}+\cdots \\
& +\mu_{e} P_{s}\left(1-P_{s}\right)+2 \mu_{e} P_{s}\left(1-P_{s}\right)^{2}+\cdots \\
= & \bar{D} . P_{s} \sum_{i=0}^{\infty}\left(1-P_{s}\right)^{i}+\mu_{e}\left(1-P_{s}\right) \sum_{j=1}^{\infty} j P_{s}\left(1-P_{s}\right)^{j-1} \\
= & \bar{D} P_{s} \frac{1}{1-\left(1-P_{s}\right)}+\frac{\mu_{e}\left(1-P_{s}\right)}{P_{s}} \\
= & \bar{D}+\frac{\mu_{e}}{P_{s}}-\mu_{e} .
\end{aligned}
$$

To validate these results, we perform simulations written in $\mathrm{C}++$, which are based on a single source-destination pair. The source and destination nodes randomly harvest energy and consume that from ambient activities. At time slot 5,000, the source generates a packet and inserts that into the transmission queue. We then record the time required to forward the packet to the destination. The simulation is repeated 10,000 times and averaged for each observation. We also take into consideration that energy might be harvested while a node is still active. This additional energy is aggregated in an energy buffer. The size of the energy buffer is assumed to be equal to $3 E_{h}$. Results provided in Figure 11 show that up to $37 \%$ improvement can be achieved through the proposed predictive retransmission scheme.

\section{PROPOSED TRANSMISSION DIVERSITY SCHEMES WITH PREDICTIVE RETRANSMISSIONS}

We now discuss the adoption of the predictive retransmission scheme to enhance the delay performance of both unicast routing with cooperative relaying and opportunistic routing.

\subsection{Cooperative Relaying with Predictive Retransmissions}

We first describe how the predictive retransmission procedure can be applied to cooperative relaying and then analyze the delay. When a transmitter's first attempt becomes unsuccessful, it calculates its optimal waiting time $T_{o}^{p}$ before the next retransmission according to its current energy. After each unsuccessful retransmission attempt, if the node is left with some energy, it finds the next optimum retransmission interval based on its current parameters. This process continues until the transmitter runs out of energy. To facilitate cooperative relaying, if any neighboring node overhears a transmission, it captures the packet and determines the intended receiver. If 


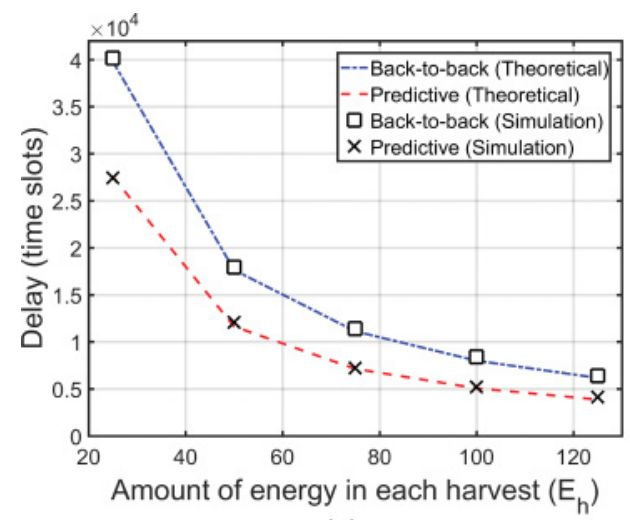

(a)

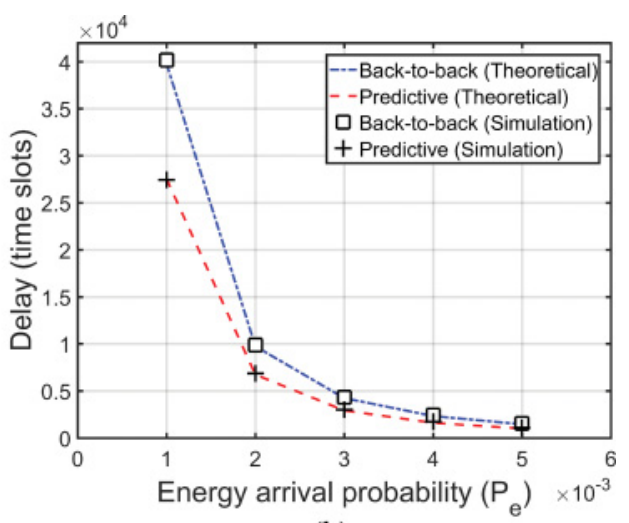

(b)

Fig. 11. Delay performance in an intermittently connected wireless link using predictive and back-to-back retransmissions.

the receiver is also within the transmission range of that node, it waits to see if the receiver acknowledges the transmission from the source. If it does not overhear the acknowledgment, then it assumes that the transmitter's attempt was unsuccessful, and it considers cooperating in forwarding the packet. The cooperator sets up its own $T_{o}^{p}$ according its energy buffer to help forward the packet.

To analyze the delay performance of the retransmission scheme with cooperative relaying, let's consider a scenario where a source and $\kappa$ other cooperators are trying to forward a packet to the receiver. At any given instance, the probability that any node is trying to forward a packet is approximately $P_{\theta 1} \approx \frac{T_{\theta}}{\mu_{e}}$, where $T_{\theta}=\left(1+T_{o}^{p}+1\right)$ for predictive retransmission and $T_{\theta}=(1+\beta)$ for back-to-back retransmissions. The probability that at least one among $(\kappa+1)$ nodes attempts to transmit the packet to the receiver can be expressed as

$$
P_{\theta 1}=1-\left(1-P_{\theta 1}\right)^{\kappa+1} \text {. }
$$

For a single node, the success probability at any random time slot is $P_{s 1}=\frac{P_{s}}{T_{\theta}}$. Therefore, the overall probability of forwarding the packet with the help of $(\kappa+1)$ nodes at each slot is $P_{s 1} P_{\theta 1}$. Hence, the expected delay to successfully deliver the packet is

$$
E\left[\operatorname{Delay}_{(\kappa+1)}\right] \approx \frac{1}{P_{s 1} P_{\theta 1}} .
$$

Numerical results obtained using the aforementioned model on the delay performance of cooperative routing over a wireless link with different number of relays are plotted in Figure 12. The results indicate that substantial performance improvement (approximately 30\%) can be achieved through the predictive retransmission policy over back-to-back retransmissions when cooperative relays are involved. Simulation results validate these results as well.

\subsection{Opportunistic Routing with Predictive Retransmissions}

Unlike unicast routing with cooperative relaying, opportunistic routing does not have a fixed (predefined) route. We propose that each transmission is forwarded by one or more nodes from a set of neighbors in the direction of the destination. Therefore, the predictive retransmission scheme in this case must be designed in such a way that at least one among the forwarder node set can be 


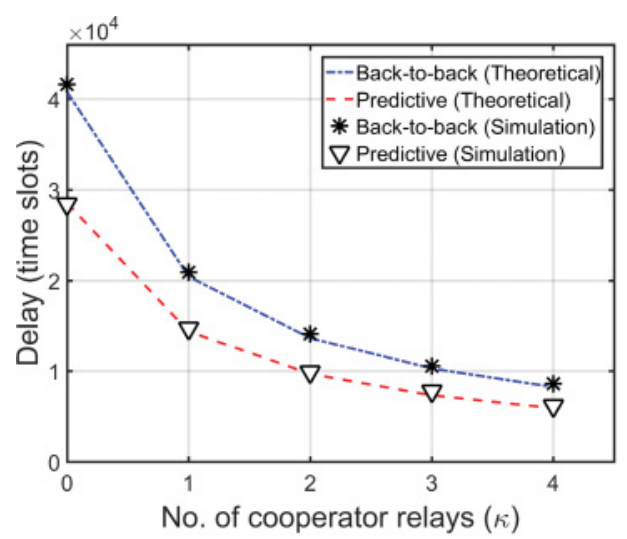

(a)

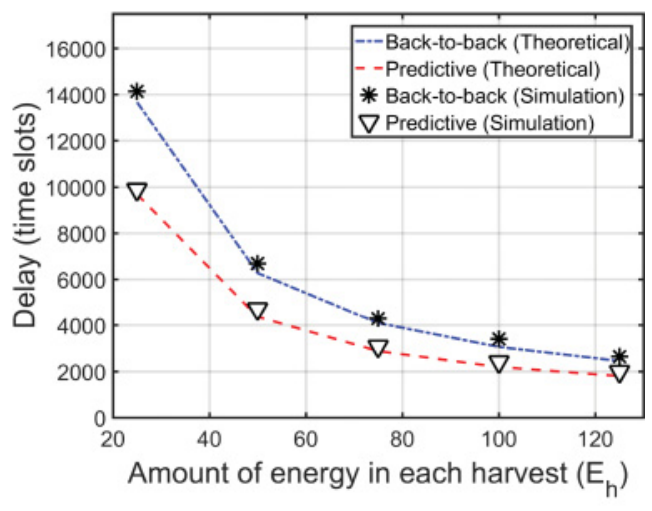

(b)

Fig. 12. Delay performance in intermittently connected wireless link using cooperative relaying with different retransmission policies.

reached. If the number of nodes in such a forwarder set is $\xi$, then the success probability would be

$$
P_{O P}=P_{w}^{t}\left(T_{w}\right)\left[1-\left(1-\sum_{x=1}^{T_{w}+1}\left[P_{e}\left(1-P_{e}\right)^{(x-1)} P_{w}^{r}\left(T_{w}+1-x\right)\right] P_{c}\right)^{\xi}\right]
$$

Therefore, once the first transmission in opportunistic routing fails, the transmitter estimates the retransmission interval according to the previoius equation and retransmits accordingly. Whenever a potential forwarder receives a data packet, it sends an acknowledgment to the source declaring its proposition as a forwarder. Since multiple nodes might receive the data packet and send acknowledgments simultaneously, they use a contention window to randomly access the channel. After receiving acknowledgments from multiple potential forwarders, the source decides who will be the next forwarder and sends a confirmation to that node to make sure that only one copy of the packet gets forwarded.

\section{SIMULATION RESULTS}

We evaluate the performance of the proposed predictive retransmission scheme with cooperative relaying and opportunistic routing using the Castalia simulator (Pediaditakis et al. 2010), which is an event-driven wireless sensor network simulator based on the OMNeT++ platform. The energy consumption profile of a sensor node is obtained from the standard MICAz mote specifications. Table 4 summarizes some of these parameters. We first consider the event-monitoring application, where one (single packet stream) or more (overlapping packet streams) node(s) transmit data packets to the sink to report an event over an intermittently connected WSN. While the single-stream scenario provides low overhearing probabilities, which is assumed in our mathematical model, the overlapping-streams scenario is used to capture the effect of correlated data traffic as well. Both grid and random node placements are considered. Next, we simulate a data collection scenario, where multiple sensors periodically transmit packets to the sink. We mainly use end-to-end latency and packet loss as performance metrics for these simulations. For a baseline comparison, we also obtain the performance using a traditional least cost (Dijkstra's shortest-path) routing protocol with back-to-back retransmissions. In all simulations, a 95\% confidence interval falls within the $3 \%$ of the presented result. 
Table 4. Simulation Parameters

\begin{tabular}{|l|c|}
\hline Total power consumed during transmission & $42.55 \mathrm{~mW}$ \\
\hline Total power consumed during reception & $73.54 \mathrm{~mW}$ \\
\hline Total power consumed during sleep & $0.105 \mathrm{~mW}$ \\
\hline Overall size of the transmission packet & $130 \mathrm{~B}$ \\
\hline Data transmission rate & $250 \mathrm{Kbps}$ \\
\hline Sleep interval & $125 \mathrm{~ms}$ \\
\hline Listen interval & $5 \mathrm{~ms}$ \\
\hline Amount of energy in each harvest & $52 \mathrm{~mJ}$ \\
\hline Energy arrival probability & 0.003 \\
\hline
\end{tabular}
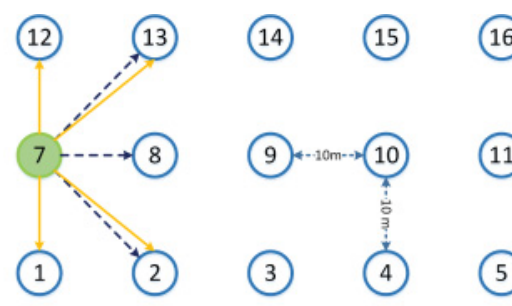

(17)

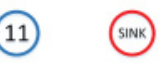

(5) (6)

(a)

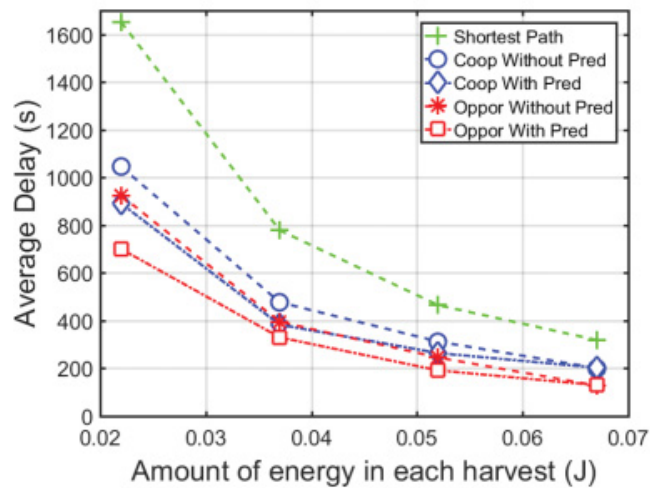

(b)

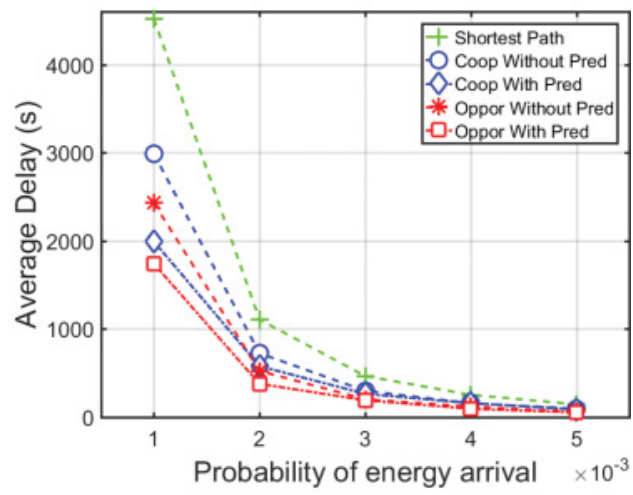

(c)

Fig. 13. Delay performance for single packet stream in event-monitoring application. (a) Node configuration for the simulation. (b) End-to-end delay at various harvested energy amounts. (c) End-to-end delay at various energy arrival probabilities.

\subsection{Event Monitoring: Single Packet Stream}

In this configuration, 18 energy-harvesting sensor nodes are placed in a uniform grid as illustrated in Figure 13(a). The node separation is assumed to be $10 \mathrm{~m}$ and the transmission radius is set to $15 \mathrm{~m}$. At a certain time, node 7 generates a packet and tries to forward it to the sink. This represents an event detection scenario for node 7 . We monitor the end-to-end latency in forwarding the packet with both cooperative relaying and opportunistic routing using predictive retransmissions. It must be noted here that each node may have different sets of forwarder nodes (opportunistic routing) or cooperator nodes (cooperative relaying) based on the chosen routing protocol. For example, in unicast routing with cooperative relaying, node 7 chooses 8 as the next hop, which allows nodes 


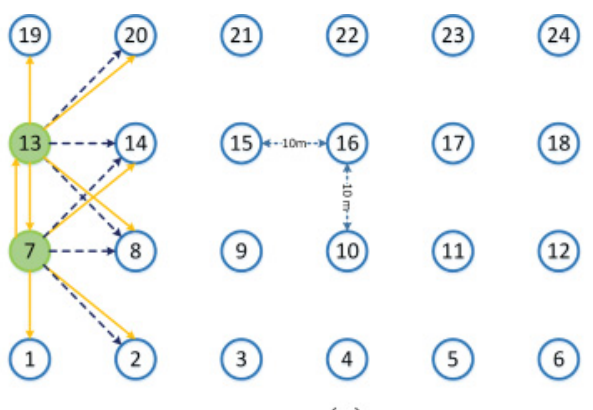

(a)

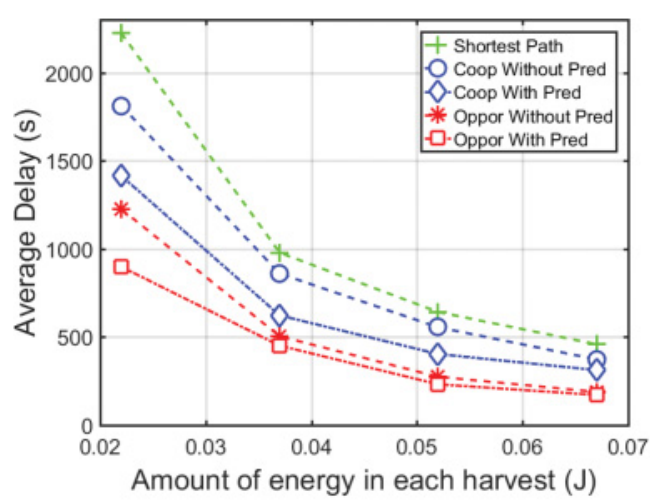

(b)

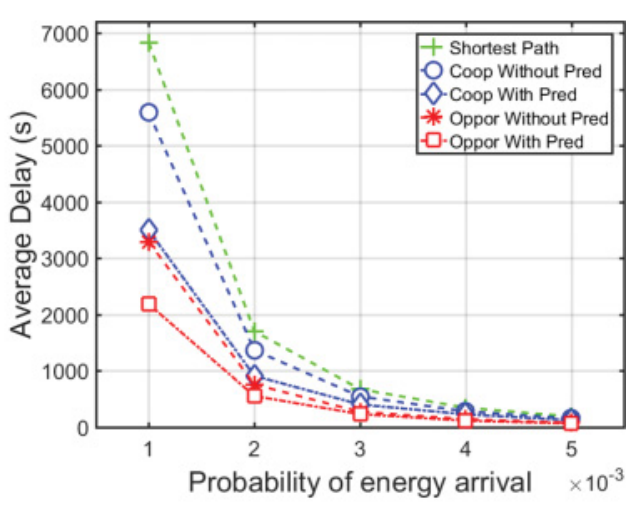

(c)

Fig. 14. Delay performance for multiple streams in event-monitoring application. (a) Node configuration for the simulation. (b) End-to-end delay at various harvested energy amounts. (c) End-to-end delay at various energy arrival probabilities.

$1,2,12$, and 13 to have the opportunity to become cooperators for 7 . When opportunistic routing is used, nodes 2, 8, and 13 can be in the forwarder set. We repeated the simulation 100 times and took the average. The results are presented in Figures 13(b) and 13(c).

It can be seen that for both cooperative and opportunistic approaches, application of the predictive retransmission scheme provides significant improvements over back-to-back repetitive retransmissions. The average delay for all of the proposed schemes is lower than that obtained using shortest-path routing, with as much as $62 \%$ delay reduction using opportunistic routing with predictive retransmissions. Also note that the primary route used in the cooperative scheme is the same as the shortest-path route. This clearly illustrates the benefits of using cooperative transmissions (i.e., transmission diversity) and the predictive retransmission scheme in reducing the end-to-end delay in the ICSN.

\subsection{Event Monitoring: Overlapping Packet Stream}

In this case, we consider a network of 25 nodes that are arranged in a uniform grid as shown in Figure 14(a). At a certain time, we assume that nodes 7 and 13 simultaneously generate packets to be transmitted to the sink. This represents the scenario where multiple nodes detect the same event and try to report it to the sink. The multihop routes of both cases are chosen using a shortest-path algorithm and might have overlaps. We record the average of the end-to-end transmission delays 


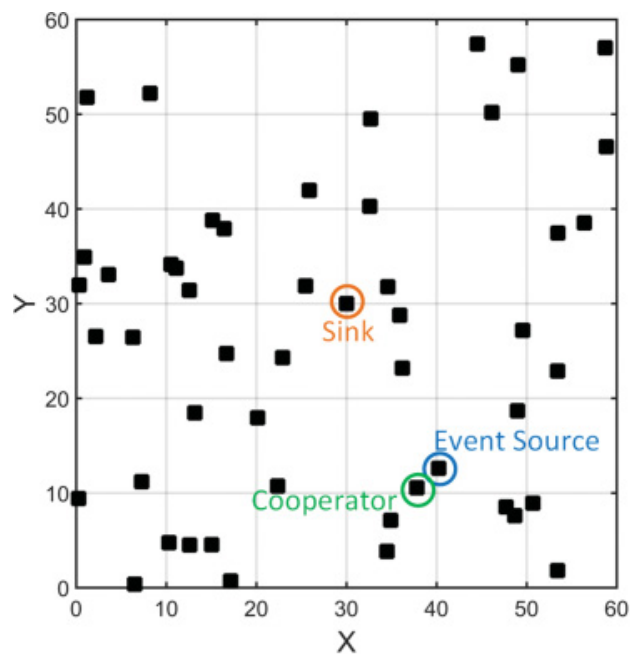

(a)

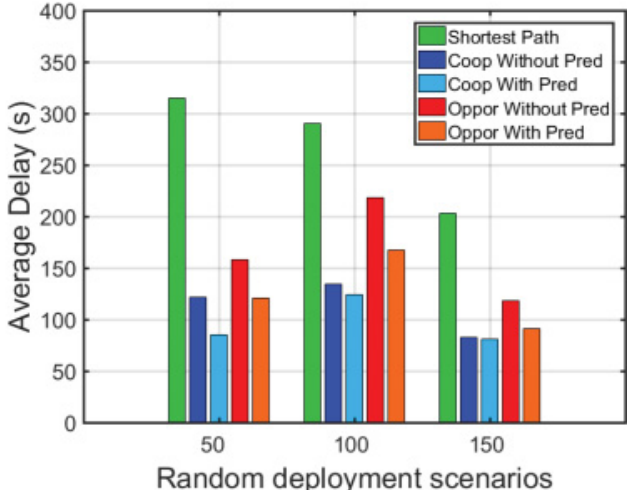

(b)

Fig. 15. Delay performance for event monitoring application with random topology. (a) Node configuration for the simulation. (b) End-to-end delay performance.

from both paths obtained from a number of simulation runs and plot the averages in Figures 14(b) and $14(\mathrm{c})$.

It is evident from Figure 14 that shortest-path routing has the highest delay. In Figure 14(c), opportunistic routing with predictive retransmissions provides a significant $68 \%$ performance gain. However, we also notice that the performance improvement using the cooperative approach is low when predictive retransmission is not applied. This is mostly due to the excessive overhearing from nearby nodes (as two routing paths might share the same set of cooperating nodes). Furthermore, no attempt has been made to eliminate unnecessary cooperation attempts (for instance, cooperators may continue to relay even after the packet is already delivered by other cooperators). In our previous work (Rajib and Nasipuri 2015), details of reducing these wasteful transmissions are described.

\subsection{Event Monitoring: Random Node Topology}

In this scenario, nodes are randomly distributed over a $60 \times 60$-meter-square region using a uniform (random) distribution, as illustrated in Figure 15(a). The sink is located at the center. We consider three different node densities with 50,100, and 150 nodes deployed over the region. For all the simulations, we chose a single-source node placed at a random location. We then record the end-to-end latency in forwarding a packet from the source to the sink with various schemes. Results obtained from simulations are presented in Figure 15(b), which show that significant improvements are achieved with the predictive retransmission scheme in these scenarios as well.

Here, one interesting observation is that sometimes cooperative relaying outperforms opportunistic routing. This is due to the fact that in opportunistic routing, most nodes do not have more than one node in the forwarder set that can provide routing progress. In cooperative relaying, however, any node within range of the transmitter and the next-hop node can relay on behalf of the transmitter, even if it is not closer to the destination. An illustration is depicted in Figure 15(a), where the source gets the cooperation from a neighbor that is the same distance as that of the 


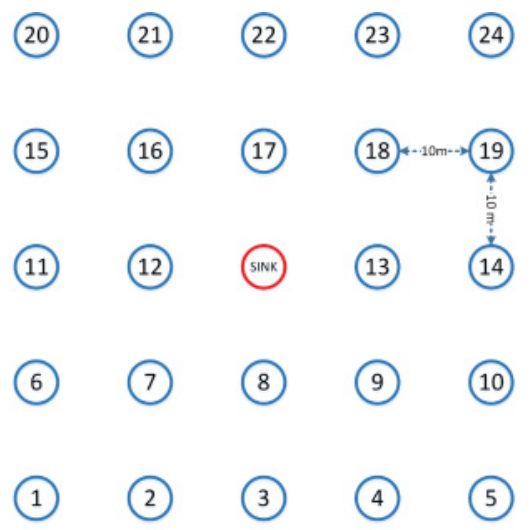

(a)

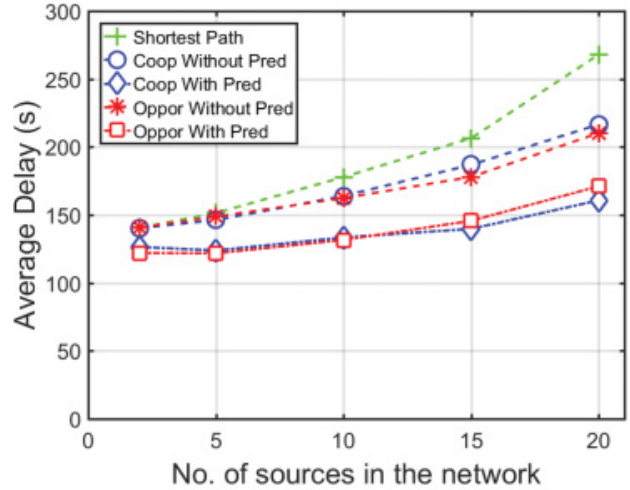

(b)

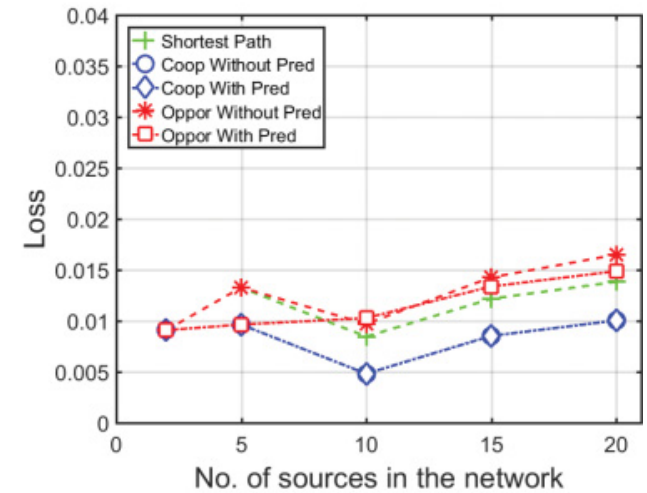

(c)

Fig. 16. Delay performance for data collection application. (a) Node configuration for the simulation. (b) Delay at various harvested energy amounts. (c) Packet loss rate with finite buffer size.

source from the destination, and would not have been involved in opportunistic routing. In this simulation setup, we achieved up to $73 \%$ gain compared to traditional shortest-path routing.

\subsection{Data Collection}

We now consider a data collection scenario where a random subset of nodes are selected as sources that periodically transmit data packets to the sink. Here, we consider that the ICSN is deployed in a uniform grid as shown in Figure 16(a). We consider the data transmission interval to be sufficiently large so as to avoid buffer overflow and congestion problems, so that the packet loss (Figure 16(c)) is low for all cases. Results presented in Figure 16(b) show that significant improvement (up to 41\%) is achieved with the proposed predictive retransmission scheme when applied to either opportunistic or cooperative routing, in comparison to shortest-path routing.

\section{CONCLUSIONS}

The problem of minimizing the transmission delay in energy-harvesting wireless sensor networks characterized by random node outages is addressed. In such networks, the key issue is to optimally use the limited energy resources in the wireless sensors in a timely manner to maximize 
the success probability of transmissions. We investigate two approaches for achieving transmission diversity in such intermittently connected networks: one that uses neighbor cooperation for forwarding packets over a predefined route, and one that opportunistically forwards packets over nodes that minimize the transmission delay. We apply an optimized retransmission scheme for both methods that determines the best transmission time based on the statistical characteristics of energy availability and usage at the nodes. Performance evaluations obtained from computer simulations demonstrate that the proposed schemes can provide as much as $73 \%$ improvement on the end-to-end transmission delay in comparison to a traditional least-cost routing approach that uses back-to-back transmissions. The proposed predictive retransmission concept contributes to over $37 \%$ reduction in the transmission delay for unicast routing with cooperative retransmissions and up to $33 \%$ reduction in the case of opportunistic routing.

\section{REFERENCES}

Anish Arora, Prabal Dutta, Sandip Bapat, Vinod Kulathumani, Hongwei Zhang, Vinayak Naik, Vineet Mittal, Hui Cao, Murat Demirbas, Mohamed Gouda, Youngri Choi, Ted Herman, Sandeep Kulkarni, Mahesh Arumugam, Mikhail Nesterenko, Adnan Vora, and M. Miyashita. 2004. A line in the sand: A wireless sensor network for target detection, classification, and tracking. Computer Networks 46, 5 (2004), 605-634.

Majid Bahrepour, Nirvana Meratnia, Mannes Poel, Zahra Taghikhaki, and Paul J. M. Havinga. 2010. Distributed event detection in wireless sensor networks for disaster management. In 2nd International Conference on Intelligent Networking and Collaborative Systems (INCOS'10). IEEE, 507-512.

Eloi Garrido Barrabes. 2016. Opportunistic Routing for Indoor Energy Harvesting Wireless Sensor Networks. Master's thesis. Delft University of Technology, the Netherlands.

Haiming Chen, Li Cui, and Victor O. K. Li. 2009. A joint design of opportunistic forwarding and energy-efficient mac protocol in wireless sensor networks. In Global Telecommunications Conference. IEEE, 1-6.

Jean-Marie Dilhac and Marise Bafleur. 2014. Energy harvesting in aeronautics for battery-free wireless sensor networks. IEEE Aerospace and Electronic Systems Magazine 29, 8 (2014), 18-22.

Zhi Ang Eu, Hwee-Pink Tan, and Winston K. G. Seah. 2010. Opportunistic routing in wireless sensor networks powered by ambient energy harvesting. Computer Networks 54, 17 (2010), 2943-2966.

Xenofon Fafoutis, Alessio Di Mauro, Charalampos Orfanidis, and Nicola Dragoni. 2015. Energy-efficient medium access control for energy harvesting communications. IEEE Transactions on Consumer Electronics 61, 4 (2015), 402-410.

Xiaofan Jiang, Joseph Polastre, and David Culler. 2005. Perpetual environmentally powered sensor networks. In 4th International Symposium on Information Processing in Sensor Networks, 2005 (IPSN'05). IEEE, 463-468.

Olaf Landsiedel, Euhanna Ghadimi, Simon Duquennoy, and Mikael Johansson. 2012. Low power, low delay: Opportunistic routing meets duty cycling. In 11th International Conference on Information Processing in Sensor Networks (IPSN'12). IEEE, 185-196.

Ying Li and Radim Bartos. 2014. A survey of protocols for intermittently connected delay-tolerant wireless sensor networks. Journal of Network and Computer Applications 41 (2014), 411-423.

Sha Liu, Kai-Wei Fan, and Prasun Sinha. 2009. CMAC: An energy-efficient MAC layer protocol using convergent packet forwarding for wireless sensor networks. ACM Transactions on Sensor Networks (TOSN) 5, 4 (2009), 29.

Masateru Minami, Takashi Morito, Hiroyuki Morikawa, and Tomonori Aoyama. 2005. Solar biscuit: A battery-less wireless sensor network system for environmental monitoring applications. In The 2nd International Workshop on Networked Sensing Systems.

David Moss, Jonathan Hui, and Kevin Klues. 2007. Low power listening. TinyOS Core Working Group, TEP 105 (2007).

Dimosthenis Pediaditakis, Yuri Tselishchev, and Athanassios Boulis. 2010. Performance and scalability evaluation of the Castalia wireless sensor network simulator. In Proceedings of the 3rd International ICST Conference on Simulation Tools and Techniques. ICST (Institute for Computer Sciences, Social-Informatics and Telecommunications Engineering), 53.

Michael Peigney and Dominique Siegert. 2013. Piezoelectric energy harvesting from traffic-induced bridge vibrations. Smart Materials and Structures 22, 9 (2013), 095019.

Md. Majharul Islam Rajib and Asis Nasipuri. 2015. Delay performance of intermittently connected wireless sensor networks with cooperative relays. In International Conference on Communication Workshop (ICCW'15). IEEE, 1994-1999.

Hossein Shafieirad, Raviraj S. Adve, and Shahram ShahbazPanahi. 2016. Large scale energy harvesting sensor networks with applications in smart cities. In Smart City 360. Springer, 215-226.

Faisal Karim Shaikh and Sherali Zeadally. 2016. Energy harvesting in wireless sensor networks: A comprehensive review. Renewable and Sustainable Energy Reviews 55 (2016), 1041-1054. 
William J. Stewart. 2009. Probability, Markov Chains, Queues, and Simulation: The Mathematical Basis of Performance Modeling. Princeton University Press.

Sujesha Sudevalayam and Purushottam Kulkarni. 2011. Energy harvesting sensor nodes: Survey and implications. IEEE Communications Surveys and Tutorials 13, 3 (2011), 443-461.

Marco Tacca, Paolo Monti, and Andrea Fumagalli. 2007. Cooperative and reliable ARQ protocols for energy harvesting wireless sensor nodes. IEEE Transactions on Wireless Communications 6, 7 (2007), 2519-2529.

Kaya Tutuncuoglu and Aylin Yener. 2011. Short-term throughput maximization for battery limited energy harvesting nodes. In 2011 IEEE International Conference on Communications (ICC'11). IEEE, 1-5.

Masaya Yoshida, Tomoya Kitani, Masaki Bandai, Takashi Watanabe, Pai H. Chou, and Winston K. G. Seah. 2012. Probabilistic data collection protocols for energy harvesting wireless sensor networks. International fournal of Ad Hoc and Ubiquitous Computing 11, 2-3 (2012), 82-96.

Received July 2016; revised April 2017; accepted April 2017 